\title{
Canadian
} Science Publishing

Canadian Journal of Physics

Revue canadienne de physique

\section{A New Class of Anisotropic Solutions of the Generalized TOV Equation}

\begin{tabular}{|r|l|}
\hline Journal: & Canadian Journal of Physics \\
\hline Manuscript ID & cjp-2016-0365.R1 \\
\hline Manuscript Type: & Article \\
\hline Date Submitted by the Author: & 07-Jun-2016 \\
\hline Complete List of Authors: & $\begin{array}{l}\text { Riazi, N.; Shahid Beheshti University, } \\
\text { Hashemi, S. Sedigheh; Shahid Beheshti University, Physics } \\
\text { Sajadi, S. Naseh; Shahid Beheshti University } \\
\text { Assyyaee, Shahrokh; Shahid Beheshti University }\end{array}$ \\
\hline Keyword: & $\begin{array}{l}\text { tov equation, relativistic polytropes, exact solutions, relativistic stars, self- } \\
\text { gravitating systems }\end{array}$ \\
\hline
\end{tabular}

SCHOLARONE

Manuscripts 


\title{
A New Class of Anisotropic Solutions of the Generalized TOV Equation
}

\author{
Nematollah Riazi; S. Sedigheh Hashemi, S. Naseh Sajadi and Shahrokh Assyyaee \\ Department of Physics, Shahid Beheshti University, G.C., Evin, Tehran 19839, Iran
}

August 18, 2016

\begin{abstract}
We present gravitating relativistic spheres composed of an anisotropic, barotropic fluid. We assume a bi-polytropic equation of state which has both linear and power-law terms. The generalized Tolman-Oppenheimer-Volkoff (TOV) equation which describes the hydrostatic equilibrium is used and the full system of equations are solved for solutions which are regular at the origin and asymptotically flat. Conditions for the appearance of horizon and a basic treatment of stability are also presented.
\end{abstract}

\section{Keywords: TOV equation, gravitating polytropes, exact solutions}

\section{Introduction}

The Lane-Emden equation is still a versatile and useful modeling device in astrophysics for describing spherical polytropes[1]. To obtain the Lane-Emden equation one starts with three essential assumptions: spherical symmetry, hydrostatic equilibrium and a polytropic equation of state [2]. The relativistic version of the Lane-Emden equation can be easily obtained [3]. The Lane-Emden equation can be derived using the polytropic equation of state in the form $P=K \rho^{1+\frac{1}{n}}$ where $n$ is called the polytropic index. The rationale for postulating such a relation between density and pressure originates from thermodynamics. In thermodynamics, a polytropic equation comes from the adiabatic condition $d Q=0$ where $d Q$ is the transferred heat. One of the immediate consequences then is $P \rho^{\gamma}=$ constant in which $\gamma=\frac{C_{p}}{C_{v}}$, the ratio of the two specific heats [4]. The isothermal and the adiabatic processes are the most important examples of a polytropic process. For a comprehensive analysis of the non-relativistic version of the LaneEmden equation and its solutions one can refer to the classic text by Clayton (1968) [5].

In general relativity, one needs to replace the classical hydrostatic equilibrium equation with its relativistic version which is known as the TOV (Tolman-Oppenheimer-Volkoff) equation $[6,7$, 8, 9]. Assuming a polytropic equation of state, the relativistic analysis becomes more tractable. Solutions should satisfy appropriate boundary conditions in order to explain various astrophysical objects from compact stars to galactic objects [10].

Another interesting application of the TOV equation is in relation with dark matter. It can be shown that the weakly interacting massive particles (WIMPs) can form clusters of non-baryonic matter which are held together by gravity. For describing the relationship between the quantities $\rho, m$ and $n$ (density of particles) for an adiabatic system of mass varying particles, besides the

*Electronic address: n_riazi@sbu.ac.ir 
relevant thermodynamic equations and using the adiabatic condition, the explicit dependence of $p(r)$ versus $\rho(r)$ can be obtained from TOV equation in the case of hydrostatic equilibrium (see [11]-[15]).

Almost all polytropic models assume an isotropic pressure. Here, we allow anisotropic pressure by assuming different tangential and radial pressure components in the energy-momentum tensor. All principal pressures (stresses) are assumed to have double-polytropic equations of state [16]. A number of authors have investigated anisotropic models (see [17]-[23]). Assuming an anisotropic stress is by no means superficial. For example, in a compact star, although the radial pressure vanishes at the surface, one still could postulate a tangential pressure to exist. While the latter does not alter the spherical symmetry, it may create some streaming fluid motions. The ReissnerNordström solution is another relativistic solution which is supported by anisotropic stresses. A charged black hole is associated with a negative radial pressure, while the tangential pressure remains positive. On galactic scales, a spherical galaxy is well described by the King-Michie family of models $[24,25]$, which involves an anisotropic pressure. This anisotropic pressure originates from the fact that the phase space distribution function of particles depends on coordinates and velocities only through the constants of motion (energy and angular momentum). If the distribution function depends on the magnitude of the angular momentum, this will lead to an anisotropic pressure, even if the system is spherically symmetric.

In what follows, we first re-derive the TOV equation for an anisotropic fluid with spherical symmetry, using the language of differential forms (next section). Our main focus in section 3 is on deriving analytical solutions with regular behavior at the center (see [26] for a comprehensive review). In section 4 we study the gravitational lensing problem for the proposed solutions. Section 5 is devoted to a study of Killing horizons and energy conditions. Section 6 addresses thermodynamics and stability considerations and the last section reflects concluding remarks.

\section{A derivation of the anisotropic TOV equation}

Although for most astrophysical cases the classical hydrostatic equation suffices, when the gravitational field becomes strong, a general relativistic version of the hydrostatic equilibrium equation becomes inevitable. If one starts from a spherically symmetric metric assuming an isotropic energy-momentum tensor one ends with the famous TOV equation [27]

$$
-\frac{d P}{d r}=\frac{G\left(\rho c^{2}+P\right)\left(M(r)+4 \pi r^{3} P\right)}{r^{2}\left(c^{2}-2 G M(r) / r\right)} .
$$

where $P$ is the pressure and $M(r)$ is the mass variable defined by

$$
M(r)=\int_{0}^{r} \rho\left(r^{\prime}\right) 4 \pi r^{\prime 2} \mathrm{~d} r^{\prime}
$$

However, we relax the assumption of isotropic pressure and let the tangential and radial pressures to be different. Let us take the following static and spherically symmetric metric for describing a gravitating relativistic sphere in Schwarzchild coordinates $\left(x^{\mu}=(t, r, \theta, \phi)\right)$

$$
\mathrm{d} s^{2}=(-1+f(r)) c^{2} \mathrm{~d} t^{2}+(1-f(r))^{-1} \mathrm{~d} r^{2}+r^{2} \mathrm{~d} \Omega^{2},
$$

where $f(r)$ is a function of the radial coordinate $r$. Introducing an orthonormal spherically symmetric basis

$$
\theta^{0}=(-1+f(r))^{1 / 2} c d t \quad, \theta^{1}=(1-f(r))^{-1 / 2} d r, \quad \theta^{2}=r d \vartheta, \quad \theta^{3}=r \sin \vartheta d \phi,
$$


and using $d \theta^{\mu}=-\omega^{\mu}{ }_{\nu} \wedge \theta^{\nu}$ for the torsion-free spacetime, the nonzero components of the connection form will be

$$
\begin{gathered}
\omega^{0}{ }_{1}=\omega^{1}{ }_{0}=\frac{-f^{\prime}(r)}{2(1-f(r))^{1 / 2}} \theta^{0}, \\
\omega^{2}{ }_{1}=-\omega^{1}{ }_{2}=\frac{(1-f(r))^{1 / 2}}{r} \theta^{2}, \\
\omega^{3}{ }_{1}=-\omega^{1}{ }_{3}=\frac{(1-f(r))^{1 / 2}}{r} \theta^{3}, \\
\omega^{3}{ }_{2}=-\omega^{2}{ }_{3}=\frac{\cot \vartheta}{r} \theta^{3} .
\end{gathered}
$$

We also have the energy-momentum conservation law in the form

$$
D * T_{\alpha}=0
$$

where $D$ stands for absolute exterior differential [28] and $T_{\alpha}$ is the energy-momentum 1-form

$$
T_{\alpha}=T_{\alpha \beta} \theta^{\beta}
$$

In our case of interest, $* T_{\alpha}$ can be written in the form

$$
* T^{\alpha}=q^{\alpha} \eta^{\alpha} .
$$

Note that there is no summation over indices in (11), and $q^{\alpha}$ and $\eta^{\alpha}$ are defined as

$$
q^{\alpha}=\operatorname{diag}\left(-\rho c^{2}, P_{r}, P_{t}, P_{t}\right)
$$

and

$$
\eta^{\alpha}=* \theta^{\alpha}
$$

where $P_{r}$ and $P_{t}$ are the radial and tangential pressures (stresses).

From the conservation law (9) one obtains

$$
d q^{\alpha} \wedge \eta^{\alpha}=-\sum_{\beta} \omega_{\beta}^{\alpha} \wedge \eta^{\beta}\left(q^{\beta}-q^{\alpha}\right)
$$

for $\alpha=1$ we have

$$
d P_{r} \wedge \eta^{1}=\omega^{1}{ }_{0} \wedge \eta^{0}\left(q^{0}-q^{1}\right)+\omega^{1}{ }_{2} \wedge \eta^{2}\left(q^{0}-q^{2}\right)+\omega^{1}{ }_{3} \wedge \eta^{3}\left(q^{0}-q^{3}\right) .
$$

Substituting for the connection forms from (5)-(8) we obtain

$$
\begin{aligned}
(1-f(r))^{1 / 2} P^{\prime}(r) \theta^{1} \wedge \eta^{1}= & \frac{-f^{\prime}(r)}{2(1-f(r))^{1 / 2}} \theta^{0} \wedge \eta^{0}\left(\rho c^{2}+P_{r}(r)\right) \\
& -\frac{(1-f(r))^{1 / 2}}{r}\left(\rho c^{2}-P_{t}(r)\right)\left(\theta^{2} \wedge \eta^{2}+\theta^{3} \wedge \eta^{3}\right) .
\end{aligned}
$$

Using the metric (3) and matter field (12), the first two components of the Einstein field equations reduce to

$$
G_{0}^{0}=G_{1}^{1}=-\frac{1}{r} f^{\prime}(r)-\frac{1}{r^{2}} f(r)
$$


which is equivalent to $P_{r}=-\rho c^{2}$. On the other hand (17) can be written as

$$
(r f(r))^{\prime}=\frac{8 \pi G}{c^{2}} \rho r^{2},
$$

where prime stands for differentiation with respect to $r$. Solving for $f(r)$ yields

$$
f(r)=\frac{b}{r}+\frac{2}{r c^{2}} \int 4 \pi G \rho r^{\prime 2} \mathrm{~d} r^{\prime},
$$

in which $b$ is the constant of integration. Although having a singularity (black hole) at the origin is among the possibilities and should be explored separately, for the moment, we omit the first singular term of the above equation. Consequently $f(r)$ reduces to

$$
f(r)=\frac{2 G M(r)}{c^{2} r},
$$

where $M(r)$ is the gravitational mass inside radius $r$ so that

$$
M(r)=\int_{0}^{r} 4 \pi r^{\prime 2} \rho d r^{\prime}
$$

Substituting for $f(r)$ and $f^{\prime}(r)$ in (16) we finally obtain

$$
-\frac{d P_{r}}{d r}=\frac{G\left(\rho c^{2}+P_{r}\right)\left(M(r) c^{2}+4 \pi r^{3} P_{r}\right)}{r^{2}\left(c^{2}-2 G M(r) / r\right)}+\frac{2\left(P_{r}-P_{t}\right)}{r},
$$

which is the generalized Tolman-Oppenheimer-Volkoff (TOV) equation allowing anisotropic pressures. It can be seen that Eq. (22) readily reduces to the standard TOV (1) when $P_{r}=P_{t}$. In what follows, we will look for solutions of the generalized TOV equation which are asymptotically Schwarzschild.

\section{Analytical solutions}

In this section, we will derive analytical solutions of the generalized TOV equation which are nonsingular and asymptotically approach the Schwarzschild spacetime at large radii. In what follows, we assume that the radial and tangential pressures obey the following barotropic equations of state $(\mathrm{EoS})$ :

$$
\begin{aligned}
& P_{r}=w \rho c^{2}+w^{\prime} \frac{\rho^{n}}{\rho_{c}{ }^{n-1}} c^{2}, \\
& P_{t}=w_{1} \rho c^{2}+w_{2} \frac{\rho^{m}}{\rho_{c}^{m-1}} c^{2},
\end{aligned}
$$

here, $\rho_{c}$ is the central density and $w, w^{\prime}, w_{1}, w_{2}$ are the dimensionless EoS parameters. These EoSs are composed of two terms. The first term is linear which is the most widely used one in astrophysics and cosmology. The second term allows a polytropic behavior which is familiar in astrophysical applications, leading to the Lane-Emden equation in the theory of stellar structure [5]. Since from Eq. (17) we have the equality $P_{r}=-\rho c^{2}$ one obtains $w=-1$ and $w^{\prime}=0$ in Eq. (23). This value for $w$ shows that we are encountering a dark-energy-like situation. Existence of negative pressure possibly originate from violation of energy conditions. However, nowadays energy condition violating matter has become much more popular in the literature (e.g. dark energy, wormhole theory, quantum effects). In particular, the existence of a strongly negative 
pressure is an essential ingredient in dark energy models. Lobo [29] presented a generalization of the gravastar picture, by considering a matching of an interior solution governed by the dark energy equation of state $w<1 / 3$, to an exterior Schwarzschild vacuum solution at a junction interface. He assumed an isotropic pressure there. The present work differs from [29] in three respects. First, the fluid pressure is assumed to be anisotropic. Second, the EoS is not assumed to be linear, and third, we do not cut off the matter density at a certain radius, thus there is no need to apply matching conditions at the surface.

The 22 and 33 components of the Einstein equations give

$$
G_{2}^{2}=G_{3}^{3}=-\frac{1}{2} f^{\prime \prime}(r)-\frac{1}{r} f^{\prime}(r) .
$$

Eqs. (17) and (25) can be simplified by introducing the new variable $u \equiv \frac{1}{r}$ which leads to

$$
\begin{gathered}
-u^{3} f_{u}+u^{2} f=\frac{8 \pi G}{c^{2}} \rho \\
u^{4} f_{u u}=-\frac{16 \pi G}{c^{4}} P_{t}
\end{gathered}
$$

respectively. Here, the subscript $u$ indicates derivative with respect to the variable $u$. Taking derivative with respect to $u$ from Eq. (26) and then multiplying by $u$ and finally using Eq. (27), one obtains

$$
\rho(u)\left(1+w_{1}\right)+w_{2} \frac{\rho^{m}(u)}{\rho_{c}^{m-1}}=\frac{1}{2} \frac{d \rho(u)}{d \ln u},
$$

which can be solved for density $\rho(r)$ :

$$
\rho(r)=\frac{\rho_{c}}{\left[\beta\left(\frac{r}{r_{0}}\right)^{2\left(1+w_{1}\right)(m-1)}-\alpha\right]^{\frac{1}{m-1}}},
$$

where

$$
\beta \equiv\left(x_{0}^{1-m}+\alpha\right), \quad x_{0}=\frac{\rho\left(r_{0}\right)}{\rho_{c}}, \quad \alpha \equiv \frac{w_{2}}{1+w_{1}}, \quad r_{0} \equiv \frac{1}{u_{0}} .
$$

Eq. (29) is the general form of the density in all of the models which will be discussed separately in the following sub-sections. At the center of the star $r=0$ the boundary condition $\rho(r)=\rho_{c}$ implies $\alpha^{2}=1$ corresponding to $\alpha= \pm 1$ if $1+w_{1}>0$.

\subsection{Case 1}

The first case corresponds to $m=\frac{3}{2}$ in Eq. (29). By substituting the values $\alpha=-1$ and $w_{2}=-2$ into Eq. (30), we obtain $w_{1}=1$. The tangential pressure for this special case becomes

$$
P_{t}=\rho c^{2}-2 \frac{\rho^{\frac{3}{2}}}{\sqrt{\rho_{c}}} c^{2} .
$$

The mass function can be obtained by performing the integration in Eq. (21)

$$
M(r)=\frac{2 \pi \rho_{c} r_{0}^{3}}{\beta^{\frac{3}{2}}}\left[\arctan \left(\sqrt{\beta}\left(\frac{r}{r_{0}}\right)\right)-\frac{\frac{r}{r_{0}}}{2 \beta\left(\beta \frac{r^{2}}{r_{0}^{2}}+1\right)}\right],
$$


and the function $f(r)$ becomes

$$
f(r)=\frac{2 G M(r)}{c^{2} r}=\frac{4 \pi G \rho_{c} r_{0}^{3}}{\beta^{\frac{3}{2}} c^{2} r}\left[\arctan \left(\sqrt{\beta}\left(\frac{r}{r_{0}}\right)\right)-\frac{\frac{r}{r_{0}}}{2 \beta\left(\beta \frac{r^{2}}{r_{0}{ }^{2}}+1\right)}\right] .
$$

The total gravitational mass of the system will be

$$
M=\lim _{r \longrightarrow \infty} M(r)=\frac{\pi^{2} \rho_{c} r_{0}^{3}}{\beta^{\frac{3}{2}}} .
$$

Therefore, the metric is asymptotically flat $(f(r \rightarrow \infty) \rightarrow 0)$. Furthermore, at the center the mass function becomes zero, as expected for a non-singular model. Thus this case satisfies the physical requirements of a non-singular relativistic gravitating system.

The metric and density functions are shown in Figures 1 and 2, respectively.

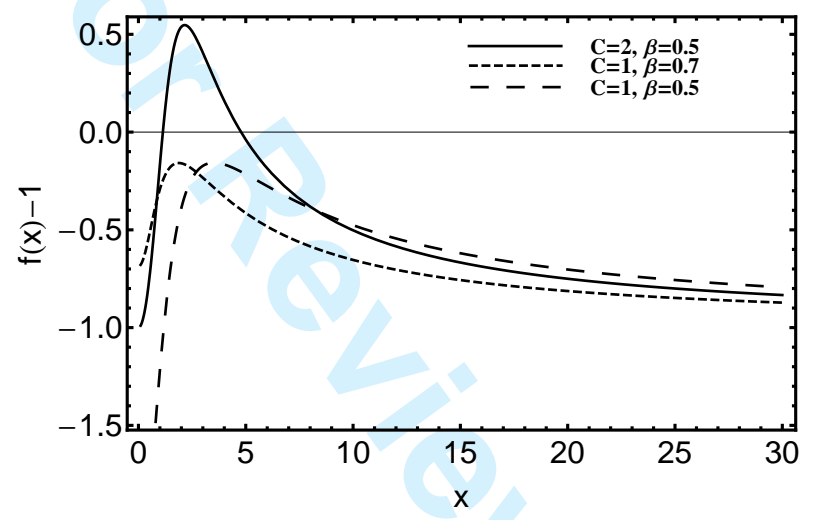

Figure 1: The metric function $g_{00}=f(x)-1$ as a function of $x \equiv r / r_{0}$ is plotted for Case $I$. Note that there are two coordinate singularities (Killing horizons) for the values $C=2$ and $\beta=0.5$. For $C=1$ and $\beta=0.7$, there are no Killing horizons. For $C=1$ and $\beta=0.5$ there are no Killing horizons and a naked singularity exists at $x=0$.

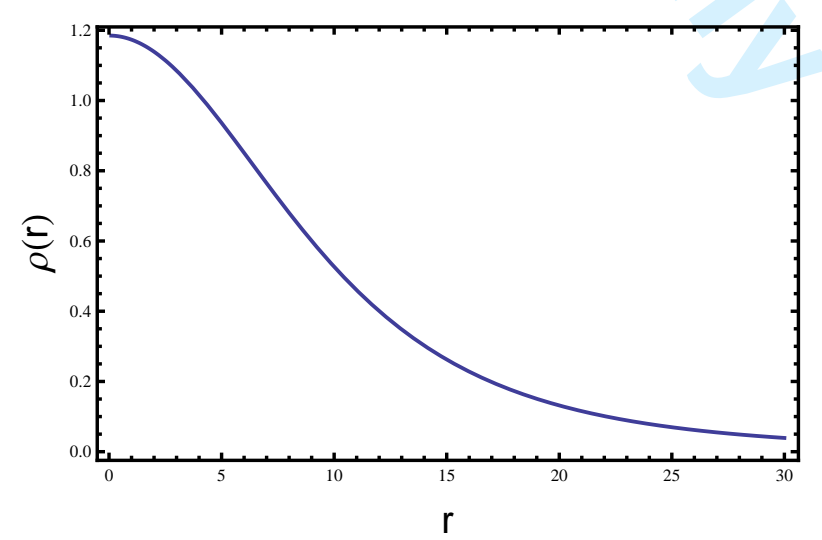

Figure 2: Energy density $\rho(r)$ as a function of radial coordinate $r$ for case 1 . 


\subsection{Case 2}

Setting $\alpha=-1$ and $w_{2}=-3$ we get $w_{1}=2$, and the tangential equation of state becomes

$$
P_{t}=2 \rho c^{2}-3 \frac{\rho^{\frac{3}{2}} c^{2}}{\sqrt{\rho_{c}}} .
$$

By substituting $w_{2}$ in Eq. (30), the mass function is obtained as

$$
M(r)=\frac{4 \pi \rho_{c} r_{0}{ }^{3}}{3} \frac{\left(\frac{r}{r_{0}}\right)^{3}}{1+\beta\left(\frac{r}{r_{0}}\right)^{3}} .
$$

Furthermore,

$$
f(r)=\frac{2 G M(r)}{c^{2} r}=\frac{8 \pi G \rho_{c} r_{0}{ }^{3}}{3 c^{2} r} \frac{\left(\frac{r}{r_{0}}\right)^{3}}{1+\beta\left(\frac{r}{r_{0}}\right)^{3}} .
$$

The total gravitational mass becomes

$$
M=\lim _{r \rightarrow \infty} M(r)=\frac{4 \pi \rho_{c} r_{0}^{3}}{3 \beta} .
$$

The metric for this case is asymptotically flat and at the center, the mass function equals zero. The metric and density functions for this case are shown in Figures 3 and 4, respectively.

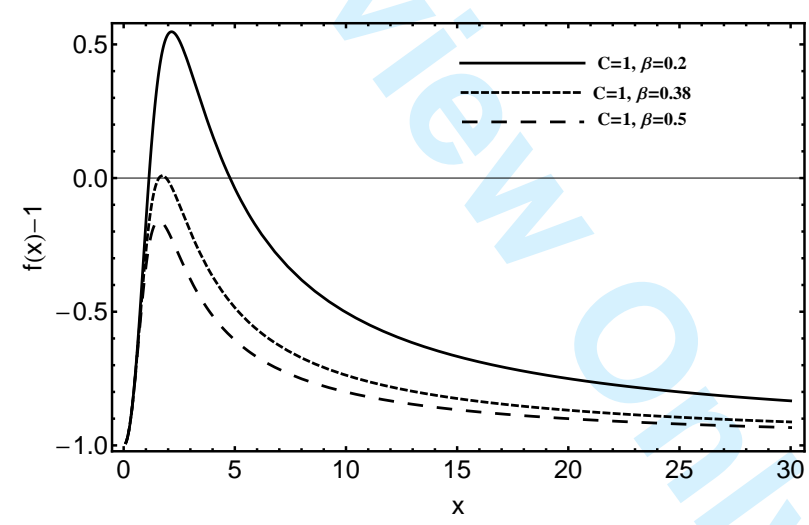

Figure 3: The metric function $g_{00}$ as a function of the dimensionless radius for case 2. For $C=1$ and $\beta=0.2$, there are two Killing horizons. For $C=1$ and $\beta=0.38$ the two Killing horizons become degenerate. Finally, with $C=1$ and $\beta=0.5$ there are no Killing horizons. This case does not have any curvature singularities. 


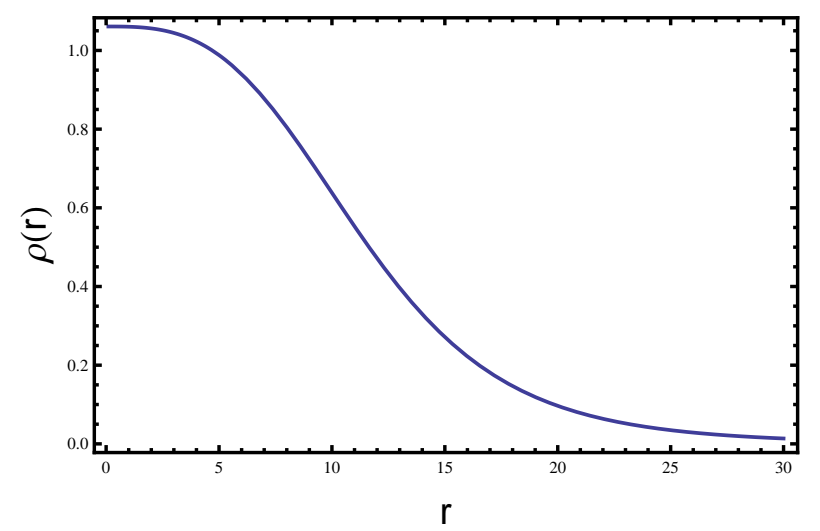

Figure 4: Energy density $\rho(r)$ as a function of radial coordinate $r$ for case 2 .

\subsection{Case 3}

Setting $\alpha=-1$ and $w_{2}=-6$ we obtain $w_{1}=5$, and the tangential equation of state becomes

$$
P_{t}=5 \rho c^{2}-6 \frac{\rho^{\frac{3}{2}} c^{2}}{\sqrt{\rho_{c}}}
$$

The mass function is obtained as

$$
M(r)=\frac{\pi \rho_{c} r_{0}^{3}}{3}\left[\frac{\left(\frac{r}{r_{0}}\right)^{3}}{1+\beta\left(\frac{r}{r_{0}}\right)^{6}}+\frac{\arctan \sqrt{\beta}\left(\frac{r}{r_{0}}\right)^{3}}{\sqrt{\beta}}\right],
$$

and

$$
f(r)=\frac{2 G M(r)}{c^{2} r}=\frac{2 \pi G \rho_{c} r_{0}{ }^{3}}{3 c^{2} r}\left[\frac{\left(\frac{r}{r_{0}}\right)^{3}}{1+\beta\left(\frac{r}{r_{0}}\right)^{6}}+\frac{\arctan \sqrt{\beta}\left(\frac{r}{r_{0}}\right)^{3}}{\sqrt{\beta}}\right] .
$$

The total gravitational mass reads

$$
M=\frac{\pi^{2} \rho_{c} r_{0}^{3}}{6 \sqrt{\beta}} .
$$

Therefore, the metric is asymptotically flat and at the center of the system the mass function vanishes. The metric and density functions for this case are shown in Figures 5 and 6 , respectively. 


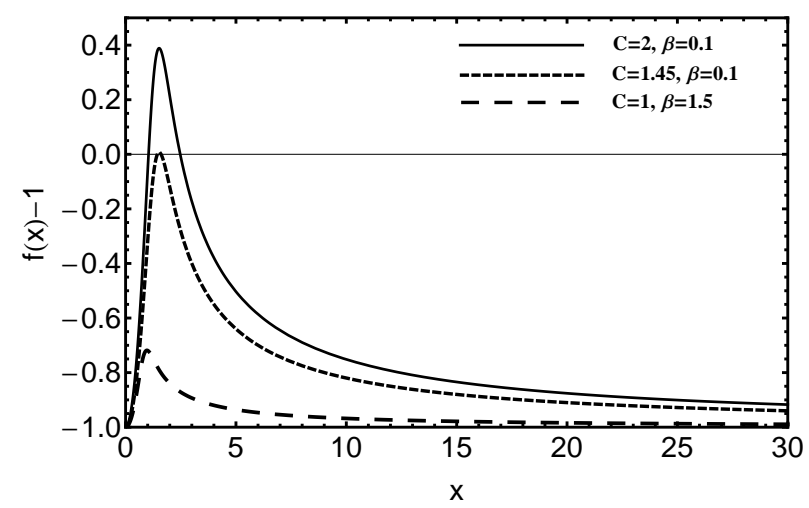

Figure 5: Two Killing horizons and a geometric singularity at $x=0$ exist for case 3 , corresponding to $C=2$ and $\beta=0.1$. $C=1.45$ and $\beta=0.1$ lead to degenerate Killing horizons. For $C=1$ and $\beta=1.5$, there are no Killing horizons but a naked singularity exists at $x=0$.

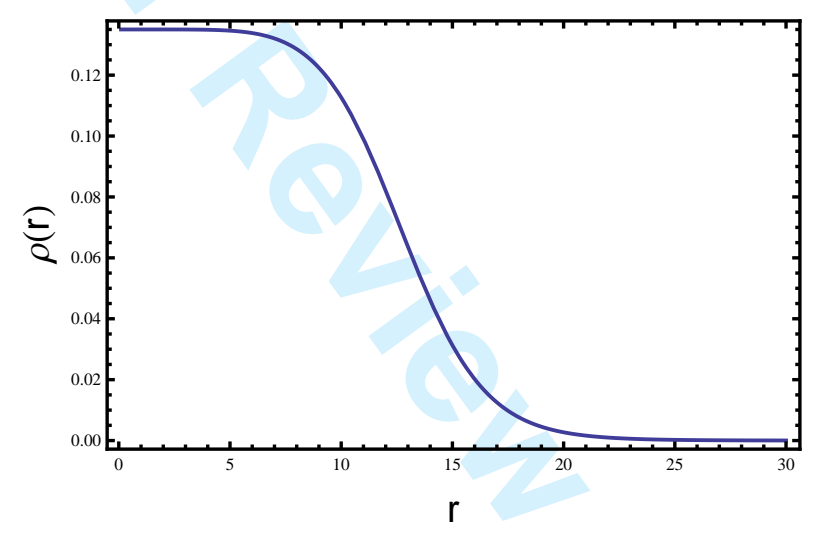

Figure 6: Energy density $\rho(r)$ as a function of radial coordinate $r$ for case 3.

\subsection{Case 4}

When $\alpha=-1$ and $w_{2}=-1$ then $w_{1}=0$ and the tangential pressures become

$$
P_{t}=-\frac{\rho^{\frac{3}{2}} c^{2}}{\sqrt{\rho_{c}}}
$$

and the mass function will be given by

$$
M(r)=\frac{4 \pi \rho_{c} r_{0}^{3}}{\beta^{2}}\left[\frac{r}{r_{0}}-\frac{2 \ln \left(\beta \frac{r}{r_{0}}+1\right)}{\beta}-\frac{1}{\beta\left(\beta \frac{r}{r_{0}}+1\right)}+\frac{1}{\beta}\right] .
$$

Moreover,

$$
f(r)=\frac{2 G M(r)}{c^{2} r}=\frac{8 \pi G \rho_{c} r_{0}^{3}}{\beta^{2} c^{2} r}\left[\frac{r}{r_{0}}-\frac{2 \ln \left(\beta \frac{r}{r_{0}}+1\right)}{\beta}-\frac{1}{\beta\left(\beta \frac{r}{r_{0}}+1\right)}+\frac{1}{\beta}\right] .
$$


However, the total mass diverges in this case.

\subsection{Case 5}

When $\alpha=-1$ and $w_{2}=\frac{3}{2}$ then $w_{1}=\frac{1}{2}$ and the tangential pressure can be expressed as

$$
P_{t}=\frac{\rho c^{2}}{2}-\frac{3 \rho^{\frac{3}{2}} c^{2}}{2 \sqrt{\rho_{c}}}
$$

The mass function will be

$$
M(r)=\frac{8 \pi \rho_{c} r_{0}^{3}}{3 \beta^{2}}\left[\frac{3}{1+\beta\left(\frac{r}{r_{0}}\right)^{\frac{3}{2}}}+\ln \left(1+\beta\left(\frac{r}{r_{0}}\right)^{\frac{3}{2}}\right)-1\right],
$$

and

$$
f(r)=\frac{2 G M(r)}{c^{2} r}=\frac{16 \pi G \rho_{c} r_{0}^{3}}{3 \beta^{2} c^{2} r}\left[\frac{3}{1+\beta\left(\frac{r}{r_{0}}\right)^{\frac{3}{2}}}+\ln \left(1+\beta\left(\frac{r}{r_{0}}\right)^{\frac{3}{2}}\right)-1\right] .
$$

In this case, too, we do not have a regular behavior at the infinity and the center, since

$$
r \longrightarrow \infty, \quad M \longrightarrow \infty \text { and } f \longrightarrow 0
$$

and

$$
r \longrightarrow 0, \quad M \longrightarrow \frac{16 \pi \rho_{c} r_{0}^{3}}{3 \beta^{2}} \text { and } f \longrightarrow \infty
$$

We have also obtained analytical solutions for $\left(w_{1}=\frac{3}{2}, w_{2}=-\frac{5}{2}\right)$ and $\left(w_{1}=3, w_{2}=-4\right)$. However, the solutions are too lengthy to be shown here.

\section{Gravitational Lensing}

In this section, we turn to the calculation of the gravitational lensing which is an important observational test to examine mass distribution in astrophysical objects. In the theory of gravitational lensing, weak lensing is an approximation which is related to small deflection angles. However, different models may differ considerably in the strong gravitational lensing regime, where the light rays pass through regions of large gravitational fields[30]-[32].

For a spherically symmetric metric (3), the deflection angle can be easily derived using the geodesics equations [33];

$$
\alpha\left(r_{m}\right)=I\left(r_{m}\right)-\pi
$$

with the total azimuthal angle $I\left(r_{m}\right)$ given by

$$
I\left(r_{m}\right)=2 \int_{r_{m}}^{\infty} \sqrt{\frac{r_{m}^{2}}{r^{2}\left(f\left(r_{m}\right)-1\right)}} \sqrt{\frac{1}{(1-f(r))\left(\frac{r^{2}}{f(r)-1}-\frac{r_{m}^{2}}{f\left(r_{m}\right)-1}\right)}} \mathrm{d} r
$$

where $r_{m}$ is the radial coordinate of the closest approach, which is literately taken to be the photon sphere. Now, to provide an example, we focus on Case 2 was discussed in section 3 . Since 
we could not find an exact solution for (52), we try the approximate method of the reference [33] and expand the denominator of (52) close to $r_{m}$. Inserting the result back into the integral one obtains

$$
I\left(r_{m}\right)=-\frac{\sqrt{2}}{4}\left[\frac{\ln \left(\frac{6\left(\beta r_{m}^{3}+r_{0}^{3}\right)^{2}}{12 \sqrt{5} \sqrt{\left(7 \beta r_{m}^{3}+17 r_{0}^{3}\right)\left(2 r_{m}^{3} \beta+5 r_{0}^{3}\right)}+\sqrt{6}\left(141 r_{m}^{3} \beta+101 r_{0}^{3}\right)}\right)}{r_{0}^{\frac{3}{2}} r_{m}^{\frac{5}{2}} \sqrt{\pi g \beta\left(2 r_{m}^{3} \beta+5 r_{0}^{3}\right)}}\right]
$$

As it is seen from Figure (7), which is plotted for the strong gravitational lensing $(53), I\left(r_{m}\right)$ demonstrates a regular behavior versus impact parameter and diverges at the photon sphere. In Figure (8) we also show the weak gravitational lensing where the light trajectory and the observer are far from the lensing object and the gravitational field is weak.

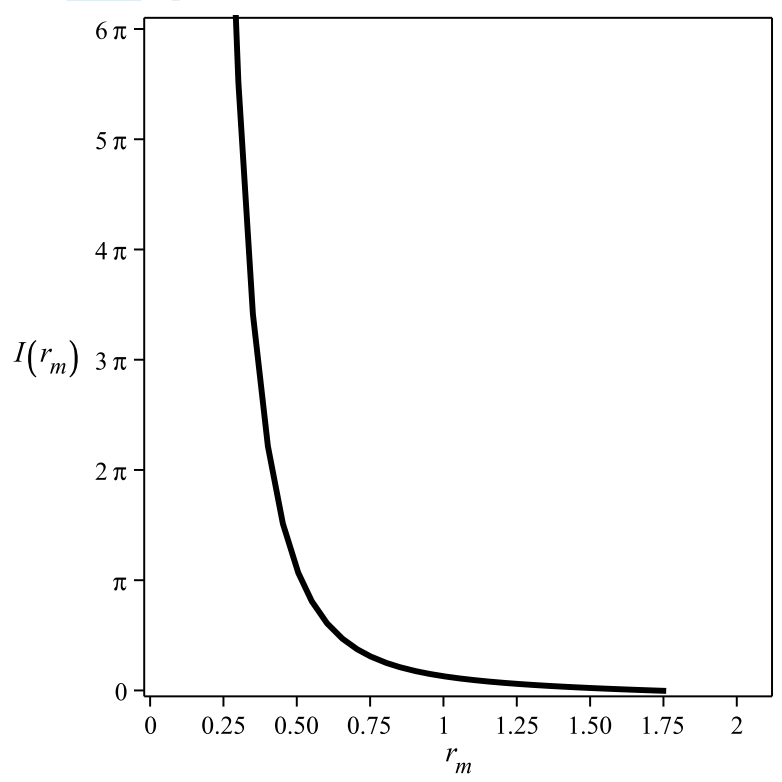

Figure 7: Total azimuthal angle with respect to the closet approach $r_{m}$ in the strong gravitational lensing regime for the choice of parameters $r_{0}$ and $\beta, g, c=1$. 


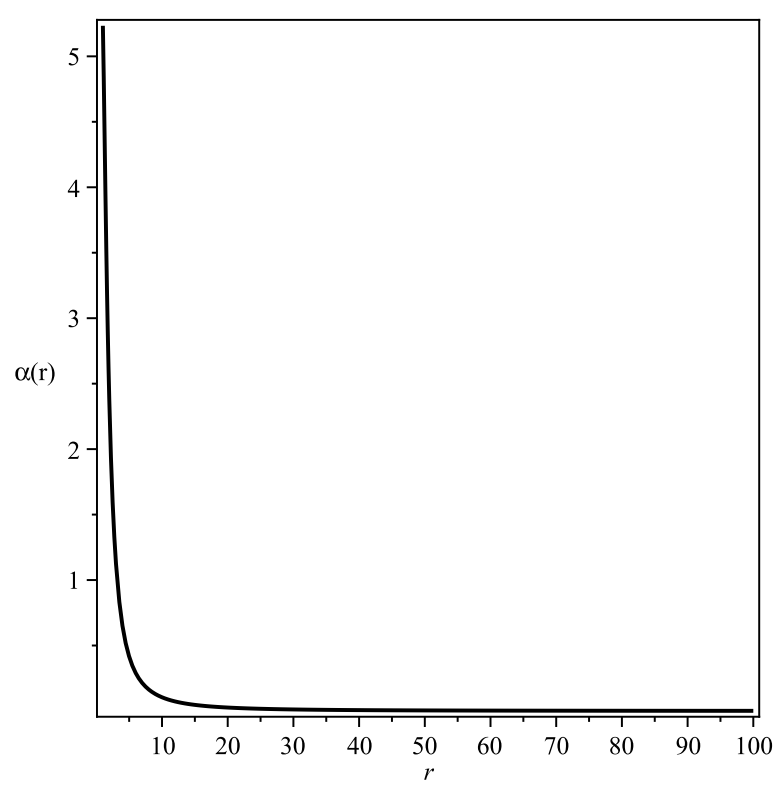

Figure 8: Deflection angle versus $r$ in the weak gravitational lensing regime which is obtained via $\alpha(r)=$ $\frac{4 M(r)}{r^{2}}$.

\section{$5 \quad$ Killing Horizons and Energy Conditions}

Since the spacetimes considered here are all static, we have the Killing vector $K=\partial_{t}$ and Killing horizon for the above cases can be obtained by putting the time-time component of the metric (3) equal to zero which gives $f\left(x_{H}\right)=1$, where $x_{H} \equiv \frac{r_{H}}{r_{0}}$. Unfortunately, analytical solutions for $r_{H}$ could not be found. Therefore, we demonstrate qualitative behavior of $f(x)-1$ for different values of $C$ and $\beta$ by plotting the cases corresponding to those of the previous section (see Figures (1)-(5). Note that we have defined $C \equiv \frac{8 \pi G \rho_{c} r_{0}^{2}}{3 c^{2}}$ for convenience.

As it can be seen in Figure (1) which is plotted for Case 1, the function $f(x)-1$ has two killing horizons for the values $C=2$ and $\beta=0.5$ with a singularity at $x=0$. For $C=1$ and $\beta=0.7$, there are no Killing horizons. For $C=1$ and $\beta=0.5$, too, there are no Killing horizons, but there is a singularity at $x=0$. Figure (3) is plotted for Case 2 for $C=1$ and $\beta=0.2$. There are two Killing horizons in this case. For $C=1$ and $\beta=0.38$, a degenerate root exists which is regarded as the extremal Killing horizon. Finally, for $C=1$ and $\beta=0.5$, there are no Killing horizons. It should be noted that this case does not have any geometrical singularity.

In Figure (5), we see that there are two Killing horizons with a singularity at $x=0$, for $C=2$ and $\beta=0.1$. $C=1.45$ and $\beta=0.1$ leads to an extremal Killing horizon. For $C=1, \beta=1.5$ we do not have any Killing horizons but there is a singularity at $x=0$.

The metric function is not plotted for cases 4 and 5, since they are not physically interesting.

Here, we examine weak (WEC) and strong (SEC) energy conditions for the cases introduced in the previous section. WEC requires $T_{\mu \nu} V^{\mu} V^{\nu} \geq 0$ for any non-spacelike vector field $V^{\mu}$ which leads to $[24]$

$$
\rho \geq 0, \quad \rho+P_{r} \geq 0, \quad \rho+P_{t} \geq 0 .
$$


SEC states that $T_{\mu \nu} V^{\mu} V^{\nu} \geq \frac{1}{2} T_{\lambda}^{\lambda} V^{\sigma} V_{\sigma}$ for any timelike vector $V^{\mu}$, or

$$
\rho+P_{r} \geq 0, \quad \rho+P_{t} \geq 0, \quad \rho+P_{r}+2 P_{t} \geq 0 .
$$

For Case 1, Eq. (54) reduces to $\rho \geq 0$ and $\rho \leq \rho_{c}$ which are both satisfied since the density is maximum at the center and the density is positive everywhere. Therefore, WEC is fully satisfied. SEC leads to $\rho_{c} \geq 4 \rho$ which is also satisfied. Thus this case satisfies the physical requirements of a realistic relativistic model.

WEC is satisfied for Case 2, similar to the previous case. SEC is also satisfied, provided that $\rho_{c} \geq \frac{9}{4} \rho$. This case also satisfies the physical properties that should be satisfied by a realistic model. For the remaining cases, the WEC is satisfied as before. SEC for Case 3, implies $\rho_{c} \geq \frac{36}{25} \rho$. However, for Case 4 we obtain $\rho \leq 0$ which contradicts positive energy theorem. Finally, for the last case, SEC requires $\rho_{c} \geq 9 \rho$.

\section{Thermodynamics and Stability Considerations}

As it was shown, there appear Killing horizons for some cases. The surface gravity $\kappa$ associated with the time-like Killing vector $K=\partial_{t}$ can be obtained via [28]

$$
\kappa^{2}=-\left.\frac{1}{4}\langle d K, d K\rangle\right|_{H}
$$

$\kappa$ can be calculated for all of the cases considered before. As an example, for the second case we have

$$
\kappa= \pm\left.\frac{1}{2} f^{\prime}(r)\right|_{r_{H}}= \pm \frac{4 \pi G \rho_{c}}{3 c^{2}}\left[\frac{2 r_{H}}{1+\beta\left(\frac{r_{H}}{r_{0}}\right)^{3}}-\frac{3 \beta r_{H}^{4}}{r_{0}^{3}\left(1+\beta\left(\frac{r_{H}}{r_{0}}\right)^{3}\right)^{2}}\right],
$$

where the horizon radius $r_{H}$ for this case can be obtained by setting $g_{00}=0$

$$
\begin{aligned}
r_{H}= & \frac{\left[\beta^{2}\left(64 m^{3} \beta-108 r_{0}^{3}+120 r_{0} \sqrt{-3 r_{0}\left(32 m^{3} \beta-27 r_{0}^{3}\right)}\right)\right]^{1 / 3}}{6 \beta} \\
& +\frac{8 m^{2} \beta}{3\left[\beta^{2}\left(64 m^{3} \beta-108 r_{0}^{3}+120 r_{0} \sqrt{-3 r_{0}\left(32 m^{3} \beta-27 r_{0}^{3}\right)}\right)\right]^{1 / 3}}+\frac{2 m}{3} .
\end{aligned}
$$

The area of the outer Killing horizon can be found straightforwardly by setting $r=r_{H}$ and $d t=d r=0$ in Eq. (3) yielding

$$
\mathrm{d} s^{2}=r_{H}^{2} \mathrm{~d} \Omega^{2} .
$$

The horizon area is then the integral of the induced volume element,

$$
A=\int \sqrt{|g|} \mathrm{d} \theta \mathrm{d} \phi
$$

so that the area of the Killing horizon is simply

$$
A=4 \pi r_{H}^{2} .
$$

Therefore, the area of the Killing horizon for all cases is given by this expression. Note that when there are two horizons, the coordinates $t$ and $r$ become spacelike and timelike, respectively, 
in the region between the two horizons. Thus the situation is somehow similar to the non-extremal Reissner-Nordstrom black hole.

We also calculate the surface gravity of a black hole by using the first law of thermodynamics which is given by[34]

$$
\kappa=8 \pi G \frac{\delta M}{\delta A},
$$

where by using equation (38), it can be easily verified that the surface gravity is the same as (57), so that the first law of thermodynamics is satisfied.

The question whether the derived solutions are stable or not requires a separate full study. However, as a first check, we have plotted the total mass versus central density for the cases 1 to 3. It can be seen from Figure (9) that the total gravitational mass has a minimum at certain central densities, signalling gross stability.

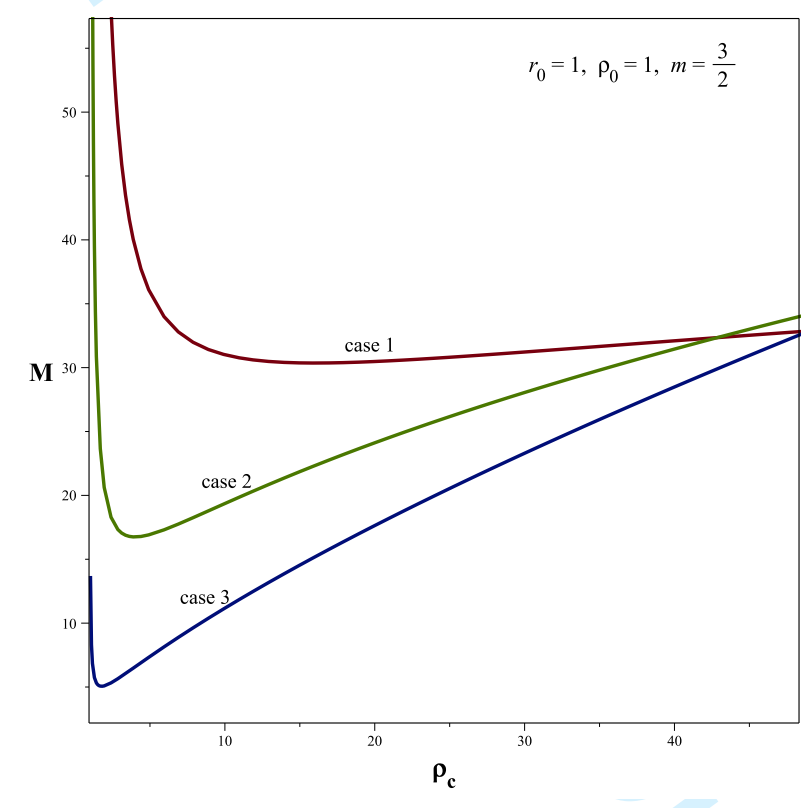

Figure 9: Total mass as a function of central density for case 1,2 and 3.

\section{Summary and Conclusion}

In this paper, we started with generalizing the TOV equation for a gravitating relativistic sphere with an anisotropic, barotropic fluid. We adapted an equation of state which has a linear term plus a power-law term which is encountered in astrophysical polytropic models. The full system of equations were then solved exactly for solutions which are regular at $r=0$ and smoothly approaching an asymptotically flat spacetime. Some singular and asymptotically non-flat cases were also mentioned, without going into details. Finally, conditions for the emergence of horizons were examined. It was shown that in most cases, it is possible to (1) avoid horizons, (2) have two horizons, and (3) have a single degenerate horizon. We calculated the deflection angle for 
the gravitational lensing of one of the cases as a function of the closest approach coordinate. The surface gravity was obtained from the first law of thermodynamics, which was shown to be the same as the one obtained from the time-like Killing vector. The status of weak and strong energy conditions were discussed, and it was shown that these conditions are satisfied in most cases. Finally, the second and third case models were shown to be stable, in the sense that the total gravitational mass is minimized for a certain central density.

\section{Acknowledgements}

Authors acknowledge the support of Shahid Beheshti University.

\section{References}

[1] L.J. Homer. The American Journal of Science and Arts. 2 50: 57, 74, (1870).

[2] S. Chandrasekhar. "An Introduction to the Study of Stellar Structure, Dover Books on Astronomy Series", Dover Publications, (2012).

[3] R.F. Tooper. Astrophysical Journal, 140, 434, (1964).

[4] R. Hakim, "Introduction to relativistic statistical mechanics". World Scientific, Singapore, (2011).

[5] D.D. Clayton. "Principles of Stellar Evolution and Nucleosynthesis". McGraw-Hill, (1968).

[6] R.C. Tolman. "Relativity, Thermodynamics and Cosmology". Oxford University Press, (1934).

[7] J.R. Oppenheimer and G.M. Volkoff. Physical Review, 55(4): 374381 (1939),

[8] R.F. Tooper, Ap.J. 140, 434, (1964).

[9] R.F. Tooper, Ap.J. 142, 1541, (1965).

[10] G.P. Horedt. "Polytropes, Applications In Astrophysics And Related Fields". Springer, (2004).

[11] A. E. Bernardini and O. Bertolami, Physical Review D, 80(12), 123011, (2009).

[12] A. E. Bernardini and O. Bertolami, Physics Letters B, 684(2), 96-100, (2010).

[13] A. E. Bernardini, Physical Review D, 80(10), 103522, (2009).

[14] M. G. Rodrigues and A. E. Bernardini, International Journal of Modern Physics D, 21(09), 1250075, (2012).

[15] A. E. Bernardini, General Relativity and Gravitation, 43(1), 245, (2011).

[16] M. Chaisi and S.D. Maharaj, Pramana J. Phys., 66, 609, (2006).

[17] R.L. Bowers and E.P.T. Liang, Ap.J. 188, 657, (1974).

[18] K. Dev and M. Gleiser, Gen. Rel. Grav. 34, 1793, (2002). 
[19] K. Dev and M. Gleiser, Gen. Rel. Grav. 35, 1435, (2003).

[20] B.V. Ivanov, Phys. Rev. D 65, 10411, (2002).

[21] M.K. Mak and T. Harko, Chin. J. Astron. Astrophys. 2, 248, (2002).

[22] M.K. Mak and T. Harko, Proc. Roy. Soc. Lond. A459, 393, (2003).

[23] L. Herrera and N.O. Santos, Phys.Rep. 286,53, (1997).

[24] S.M. Carroll. "Spacetime and Geometry. An introduction to general relativity". (2004).

[25] I.R. King. Astronomical Journal, 71, 64, (1966).

[26] L. Herrera and N. O. Santos. Physics Reports, 286(2), 53, (1997).

[27] E. Poisson and C. Will. "Gravity: Newtonian, Post-Newtonian, Relativistic", Cambridge University Press, (2014).

[28] N. Straumann "General Relativity with Applications to Astrophysics", Springer, (2004).

[29] F.S.N. Lobo, Class. Quantum Grav. 23, 1525, (2006).

[30] P. Schneider, J. Ehlers and E. E. Falco, (Springer-Verlag, Berlin), (1992).

[31] V. Perlick, Living Rev. Relativity 7, 9, (2004).

[32] M. Bartelmann, Class. Quant. Grav. 27, 233001, (2010).

[33] V. Bozza, General Relativity and Gravitation 42.9, 2269, (2010).

[34] S. Carlip, International Journal of Modern Physics D, 23(11), 1430023, (2014). 